\title{
Development and Validation of a Skin Fold Thickness Prediction Equation for Asian Indians Using Hydrodensitometry
}

\author{
Shenoy $\mathrm{S}^{*}$, Shrivastava $\mathrm{S}^{1}$, Sandhu $\mathrm{JS}^{1}$, Malhotra $\mathrm{B}^{1}$ and Gupta $\mathrm{SK}^{2}$ \\ ${ }^{1}$ Department of Sports Medicine and Physiotherapy, Guru Nanak Dev University, Amritsar, India \\ ${ }^{2}$ Indian Institute of Statistics, New Delhi, India
}

*Corresponding author: Shenoy S, Associate Professor, Department of Sports Medicine and Physiotherapy, Guru Nanak Dev University, Amritsar, India 143001, E-mail: drshweta.sportsmed@gmail.com

Citation: Shenoy S, Shrivastava S, Sandhu JS, Malhotra B, Gupta SK (2016) Development and Validation of a Skin Fold Thickness Prediction Equation for Asian Indians Using Hydrodensitometry. J Obes Overweig 2(1): 102. doi: 10.15744/2455-7633.2.102

Received Date: June 24, 2015 Accepted Date: February 23, 2016 Published Date: February 25, 2016

\begin{abstract}
Skin fold thickness prediction equations are widely used for estimating body fat percentage and thereby obesity. Equations to estimate body fat percentage for the Asian Indian population do not yet exist. The main purpose of this study was to develop and validate a new prediction equation for the estimation of body fat percentage in Asian Indian adults using anthropometric measurements (skin folds, circumference, bone breadth) and DEXA using hydro static under water weighing (hydrodensitometry) as a gold standard.
\end{abstract}

Method: 137 healthy people (77 males and 60 females), in the age group of 30-60 years were included in the study. With hydrodensitometry, anthropometric measurements such as height, weight, 10 skin folds thickness sites, 5 sites of circumference, 5 sites of bone breadths and DEXA scans were taken as variables. A linear regression model was then created for both males and females, with body fat \% calculated by hydrodensitometry used as a dependent variable and other anthropometric measurements and DEXA results used as independent variables.

Results: 4 new body fat estimation models, 2 for males and 2 for females were obtained for Asian Indians with R2 of 0.90 and 0.71 for males and 0.90 and 0.72 for females respectively. Conclusion: The new prediction equations for body fat \% estimation, which were derived and internally validated in an adult Indian population revealing a lower error difference than previously developed models.

Keywords: Obesity; Asian Indians; New predicted equation; Hydrodensitometry; DEXA

\section{Background}

There is increasing evidence that the associations between body mass index (BMI), body fat percentage (body fat \%) and body fat distribution differ across populations [1]. Particularly in the Asian population, a specific BMI reflects a higher percentage of body fat than in white or European populations [1]. Obesity is defined as an excessive amount of adipose tissue with body fat $\% \geq 25 \%$ in males, $\geq 32 \%$ in females [2]. BMI which is essentially weight $(\mathrm{kg}) /$ height $^{2}\left(\mathrm{~m}^{2}\right)$, is a measure of relative weight and is an acceptable proxy for thinness and fatness and has been directly related to health risks and death rates in many populations [1]. Thus BMI is often used as a surrogate measure of obesity, because of the difficulties in accurately estimating body fat \%, though BMI fails to distinguish between body fat and lean mass. Considering the relation of BMI cut offs with health risks, the cut off values for obesity using BMI were established as BMI $\geq 25 \mathrm{~kg} / \mathrm{m}^{2}$ (WHO) [1] and for Asians was recently revised from $25 \mathrm{~kg} / \mathrm{m}^{2}$ to $23 \mathrm{~kg} / \mathrm{m}^{2}$ in adults [3]. A meta-analysis of 32 different samples comprising a total of 31,968 patients revealed that the commonly used BMI cut off values to diagnose obesity failed to identify half of the people with excess PBF [4,5]. Recently a study by Zeng et al. 2012 [5] demonstrated that percent body fat was a better predictor of cardiovascular risk factors than BMI in a Chinese population.

Considering the global burden of obesity and its co morbidities, emphasis is now shifting towards a preventive approach as well as risk stratification for the identification of high risk individuals and lifestyle changes [6,7]. Because obesity is a common denominator in the development of lifestyle disorders, accurately calculating body fat $\%$ using field methods has now become a necessity. Several models such as Durnin and Womersley 4 sites, Jackson and Pollock 3 sites and 4 sites equation, BMI body fat \%, Garcia equation are widely used for the estimation of body fat \%, as convenient methods. Several studies have shown that these models sometimes underestimate [8,9] or overestimate body fat [10] in different populations and these models have not been validated for use in the Indian population. Considering the above factors, we derived a new prediction equation for the estimation of body fat \% using skinfold measurement, as well as common kinanthropometric measurements using hydrodensitometry as a gold standard [11,12]. We also explored the use of DEXA in the evaluation of body fat, as well bone mineral density (BMD) for evaluation of significance and possible inclusion in the equation. 


\section{Methodology}

137 healthy subjects 77 males and 60 females were included in the study within the age group of 30-60 years. The study was conducted in the months of May-November 2012. The procedure was explained and an informed consent letter was obtained from the patients before performing the test. The study was approved by the Institutional Ethics Committee. Body weight was measured by a weighing machine in kilograms (kgs). Height was measured by stadiometer without foot wear and BMI was calculated by weight $(\mathrm{kg}) /$ height $^{2}\left(\mathrm{~m}^{2}\right)$. Skinfolds measurements were taken from the right side using Harpenden skinfold caliper (Harpenden Ltd, England, United Kingdom) and sites involved biceps, triceps, subscapularis, chest, abdominal, suprailliac, thigh, knee, calf and chin. Circumference of waist, hip, thigh, calf and arm were measured with a soft tape measure to the nearest $0.1 \mathrm{~cm}$. Breadths (chest, elbow, knee, ankle and wrist) were measured using widespread vernier caliper (Holtain Ltd, Croswell, Crymch, United Kingdom). Each anthropometric site was measured using the techniques described in the Anthropometric Standardization Reference Manual [13].

Durnin and Womersley 1973 [14], explains why the Logarithm of each skinfold thickness equation was taken rather than actual measurement reasons are as follows:

Firstly, frequency distribution of most of skin fold measurement in general population is skewed, with a long tail of high readings (although this may well represent a pathological state of common obesity).

Secondly, the relationship of body density to skin folds may not be rectilinear because of a larger portion of body fat which is deposited subcutaneously with increasing obesity [14].

\section{Hydrostatic under-water weighing}

Body fat percentage was assessed using the hydrostatic underwater weighing machine" Vacumed Turbo fit 5.10" (www.vacumed. com) as described in a previous study from this laboratory [2]. Procedure was explained and participants practiced before taking the final readings. An average of three readings was taken as the final reading. The software estimated the residual lung volume using the following equations:

Male residual lung volume $=$ Vital Capacity $\times 0.24$

Female residual lung volume $=$ Vital Capacity $\times 0.28$

The \% BODY FAT was then estimated by the equation of Brozek et al. [15].

\section{DEXA}

DEXA Hologic Discovery Wi was used as a non-invasive technique for whole body and segmental fat-, bone- and lean- mass and bone density [16]. The Hologic Discovery Wi with fan beam features (Hologic) and array mode was used for all DXA scans. The characteristics and physical concepts of DXA measurements have been described previously elsewhere [17]. The scans were analyzed with the most recent software APEX version 4.0. The scan measurements and analyses were conducted following standard 9 analysis protocol as described in the Hologic User Manual. All scans were subsequently analyzed by a single trained investigator [18].

\section{Statistical analysis}

The whole data set consisted of 137 subjects with 77 males and 60 females. The data set was then randomly divided into a prediction and validation set in a ratio of 75:25 (i.e. data of randomly selected 55 males and 49 females were used to predict the model and remaining 33 subjects i.e. 22 males and 11 females were used to validate the model). The descriptive statistics of both males and females along with its significance status by one-way sample t test were used to summarize the continuous variables. Apart from descriptive statistics, ANOVA, Pearson correlation and multiple linear regression was done to create a model.

\section{Results}

137 healthy subjects of age group of 30-60 years, both male and female were included in the study. Descriptive statistics summarizing the continuous variables along with its significance are shown in (Table 1 and 2). All the variables which were found to be significant by the application of one way sample $t$ test were then put into the multiple linear regression in SPSS version 20.0 which showed all the variables in our models, it also showed the sets of variables for several models at once, by step wise and backward methods of linear regression. We found 2 best models for both males and females by these methods. Model summary are described in table 3 for both males and females. Table 3 shows that the R Square for males is 0.90 (model 1 ) and 0.71 (model 2); and for female R Square is 0.90 (model 1) and 0.717 (model 2). The error of estimate for body fat calculation for males is 2.85 (model 1) and 3.24 (model 2) and for females it is 2.24 (model 1) and 2.74 (model 2). A Table 4 indicates the results of analysis of variance (ANOVA). In these tables considering the two sums of squares introduced in class - the regression and residual (or error) sums of squares indicates that the variance of the residuals (or errors) is the value of the mean square error (MSE) which is 8.26 and 10.47 for model 1 and 2 respectively in males and 5.84 and 7.5 for model 1and 2 respectively for females. These values show that the error rate is less in model 1 in both males and females. This table also summarizes results of the $F$ test. This tests the hypothesis that the predictor (here our only predictor) shows no relationship to Y scores. The value of the test for our data is $\mathrm{F}$ is 
6.39 and 63.21 for model 1 and 2 respectively for males and 6.4 and 27.87 for model 1 and 2 respectively for female 's. The table shows us this is significant $(\mathrm{p}<.001)$. As the value of $\mathrm{F}$ is both large and small, we determine that our predictor of body fat $\%$ outcome is related to body fat $\%$ in our population.

\begin{tabular}{|c|c|c|}
\hline VARIABLES & MALES $(\mathrm{N}=77)$ & FEMALES $(\mathrm{N}=60)$ \\
\hline Age & $42.74 \pm 9.15(30,60)^{\star}$ & $43.37 \pm 8.21(30,60)^{*}$ \\
\hline Height (In Mts) & $1.69 \pm 0.008(1.53,1.88)^{*}$ & $1.55 \pm 0.05(1.45,1.69)^{*}$ \\
\hline Weight (In Kg) & $75.65 \pm 12.8(45,108)^{*}$ & $65 \pm 9.84(46,88)^{*}$ \\
\hline Skf Chin (In Mm) & $9.94 \pm 2.71(3.8,17.6)^{*}$ & $10.7 \pm 2.66(5.2,19)^{*}$ \\
\hline Skf Biceps (In Mm) & $9.36 \pm 4.17(2.4,22)^{*}$ & $12.5 \pm 3.8(3.2,23.8)^{*}$ \\
\hline Skf Triceps (In Mm) & $14.86 \pm 4.91(4.1,27.2)^{*}$ & $20.19 \pm 5.5(7.2,35.6)^{*}$ \\
\hline Skf Subscapular (In Mm) & $21.59 \pm 7.45(5.4,39.8)^{*}$ & $25.08 \pm 6.74(6.2,48.2)^{*}$ \\
\hline Skf Chest (In Mm) & $18.31 \pm 8.4(3.5,38)^{*}$ & $25.76 \pm 12.27(3.6,56)^{*}$ \\
\hline Skf Abdominal (In Mm) & $27.04 \pm 11.26(5.4,61.8)^{*}$ & $30.48 \pm 9.56(11,54) *$ \\
\hline SkfSuprailiac (In Mm) & $19.48 \pm 10.23(3.8,55.2)^{*}$ & $29.3 \pm 9.43(5,50)^{*}$ \\
\hline Skf Thigh(In Mm) & $22.33 \pm 9.21(4.5,43)^{*}$ & $27.04 \pm 9.01(8,44)^{*}$ \\
\hline Skf Knee (In Mm) & $11.36 \pm 5.66(4,33)^{*}$ & $12.7 \pm 6.13(1.08,30)^{*}$ \\
\hline Skf Calf (In Mm) & $17.45 \pm 6.65(4,35)^{*}$ & $22.02 \pm 7.16(2.2,37)^{*}$ \\
\hline Log Chin & $0.98 \pm 0.124(0.57,1.24)^{\star}$ & $1.02 \pm 0.1(0.72,1.28)^{*}$ \\
\hline Log Biceps & $0.93 \pm 0.197(0.38,1.34)^{\star}$ & $1.08 \pm 0.15(0.5,1.38)^{*}$ \\
\hline Log Triceps & $1.14 \pm 0.16(0.61,1.43)^{*}$ & $1.29 \pm 0.13(0.86,1.6)^{*}$ \\
\hline Log Subscapular & $1.30 \pm 0.17(0.73,1.6)^{*}$ & $1.38 \pm 0.13(0.8,1.68)^{*}$ \\
\hline Log Chest & $1.20 \pm 0.24(0.54,1.57)^{*}$ & $1.35 \pm 0.25(0.55,1.74)^{*}$ \\
\hline Log Abdominal & $1.39 \pm 0.20(0.73,1.79)^{*}$ & $1.46 \pm 0.14(1.041,1.732)^{*}$ \\
\hline Log Suprailiac & $1.23 \pm 0.24(0.58,1.74)^{*}$ & $1.44 \pm 0.165(0.7,1.7)^{*}$ \\
\hline Log Thigh & $1.307 \pm 0.2(0.65,1.6)^{*}$ & $1.40 \pm 0.174(0.90,1.64)^{*}$ \\
\hline Log Knee & $1.01 \pm 0.182(0.60,1.52)^{\star}$ & $1.04 \pm 0.25(0.33,1.48)^{*}$ \\
\hline Log Calf & $1.20 \pm 0.195(0.94,0.60)^{*}$ & $1.31 \pm 0.19(0.34,1.6)^{*}$ \\
\hline Arm Circumference (In Cm) & $29.65 \pm 2.9(21.2,37.5)^{*}$ & $28.45 \pm 2.94(20,55)^{*}$ \\
\hline Waist Circumference (In Cm) & $94.09 \pm 10.37(62.5,117.5)^{*}$ & $91 \pm 9.81(70,115)^{*}$ \\
\hline Hip Circumference (In Cm) & $96.04 \pm 7.37(75,112.5)^{*}$ & $98.9 \pm 11.51(40,121.25)^{*}$ \\
\hline Thigh Circumference (In Cm) & $48.58 \pm 5.62(37.5,60)^{*}$ & $45.63 \pm 7.12(15,57.5)^{*}$ \\
\hline Calf Circumference (In Cm) & $34.25 \pm 3.58(25,42.5)^{*}$ & $33.26 \pm 4.13(10,39)^{*}$ \\
\hline Bone Breadth Elbow (In Cm) & $13.81 \pm 1.78(7.5,17.5)^{\star}$ & $12.09 \pm 2.5(6.25,20)^{*}$ \\
\hline Bone Breadth Wrist (In Cm) & $8.25 \pm 1.27(6.3,12.5)^{*}$ & $6.97 \pm 1.40(5,11.25)^{*}$ \\
\hline Bone Breadth Chest (In Cm) & $50 \pm 5.2(37.5,63.8)^{*}$ & $49.24 \pm 5.96(40,71.75)^{*}$ \\
\hline Bone Breadth Knee (In Cm) & $18.8 \pm 3.08(6,23.8)^{*}$ & $16.75 \pm 2.3(12.5,21.25)^{*}$ \\
\hline Bone Beadth Ankle (In Cm) & $12.036 \pm 1.44(8.8,15)^{*}$ & $10.8 \pm 1.26(7.5,13.75)^{*}$ \\
\hline
\end{tabular}

'*' Shows that $\mathrm{P}$ value is $<0.001$ which is highly significant for all the variables.

Table 1: Descriptive statistics for both males and females

\begin{tabular}{|c|c|c|c|c|}
\hline \multirow[t]{2}{*}{ VARIABLE } & \multicolumn{2}{|c|}{ MALE } & \multicolumn{2}{|c|}{ FEMALE } \\
\hline & PREDICTION SET & VALIDATION SET & PREDICTION SET & VALIDATION SET \\
\hline & $\mathrm{N}=\mathbf{5 5}$ & $\mathrm{N}=\mathbf{2 2}$ & $\mathrm{N}=49$ & $\mathrm{~N}=11$ \\
\hline Age & $42.02 \pm 8.39^{*}$ & $44.55 \pm 10.83^{*}$ & $43.06 \pm 7.64^{*}$ & $44.64 \pm 10.75^{\star}$ \\
\hline Height (M) & $1.698 \pm 0.08^{*}$ & $1.68 \pm 0.077^{*}$ & $1.56 \pm 0.55^{*}$ & $153.91 \pm 4.57^{*}$ \\
\hline Weight (Kg) & $76.86 \pm 13.19^{*}$ & $72.64 \pm 11.36^{*}$ & $64.1 \pm 10.34^{*}$ & $69 \pm 6.12^{*}$ \\
\hline Chin Skf & $9.95 \pm 2.63^{*}$ & $9.9 \pm 2.97^{\star}$ & $10.81 \pm 2.53^{*}$ & $10.2 \pm 3.27^{\star}$ \\
\hline Biceps Skf & $9.32 \pm 4.01^{*}$ & $9.4 \pm 4.64^{*}$ & $12.40 \pm 4.04^{*}$ & $13.32 \pm 2.54^{\star}$ \\
\hline Triceps Skf & $14.51 \pm 5.23^{*}$ & $15.71 \pm 3.98^{*}$ & $20.40 \pm 5.5^{*}$ & $19.21 \pm 5.52^{*}$ \\
\hline Subscapular Skf & $21.5 \pm 7.77^{*}$ & $21.76 \pm 6.78^{*}$ & $24.86 \pm 7.13^{*}$ & $26.04 \pm 4.74^{*}$ \\
\hline
\end{tabular}




\begin{tabular}{|c|c|c|c|c|}
\hline \multirow[t]{2}{*}{ VARIABLE } & \multicolumn{2}{|c|}{ MALE } & \multicolumn{2}{|c|}{ FEMALE } \\
\hline & PREDICTION SET & VALIDATION SET & PREDICTION SET & VALIDATION SET \\
\hline & $\mathrm{N}=55$ & $\mathrm{~N}=\mathbf{2 2}$ & $\mathrm{N}=49$ & $\mathrm{~N}=11$ \\
\hline Chest Skf & $18.57 \pm 8.38^{*}$ & $17.65 \pm 8.59^{*}$ & $26.42 \pm 11.79^{*}$ & $22.79 \pm 14.44^{*}$ \\
\hline Abdominal Skf & $28.51 \pm 11.81^{*}$ & $23.39 \pm 8.99^{*}$ & $30.95 \pm 9.52^{*}$ & $28.35 \pm 9.92^{*}$ \\
\hline Suprailiac Skf & $21.02 \pm 10.86^{*}$ & $15.62 \pm 7.31^{*}$ & $30.08 \pm 9.84^{*}$ & $25.87 \pm 6.68^{*}$ \\
\hline Thigh Skf & $22.22 \pm 9.20^{*}$ & $22.61 \pm 9.44^{*}$ & $28 \pm 8.33^{*}$ & $22.71 \pm 11^{*}$ \\
\hline Knee Skf & $11.14 \pm 5.8^{*}$ & $11.89 \pm 5.37^{*}$ & $12.87 \pm 5.91^{*}$ & $11.95 \pm 7.23^{*}$ \\
\hline Calf Skf & $17.76 \pm 6.82^{\star}$ & $16.67 \pm 6.29^{*}$ & $22.07 \pm 7.03^{*}$ & $21.81 \pm 8.13^{*}$ \\
\hline Arm Circumference & $29.93 \pm 2.9^{*}$ & $28.97 \pm 2.89^{*}$ & $28.33 \pm 3.03^{*}$ & $28.98 \pm 2.61^{*}$ \\
\hline Waist Circumference & $95.17 \pm 10.41^{*}$ & $91.4 \pm 10.01^{*}$ & $91.19 \pm 10.31^{*}$ & $93.3 \pm 7.8^{*}$ \\
\hline Hip Circumference & $96.8 \pm 7.5^{*}$ & $94.15 \pm 6.95^{*}$ & $97.76 \pm 12.28^{*}$ & $103.86 \pm 4.92^{*}$ \\
\hline Thigh Circumference & $48.81 \pm 5.5^{\star}$ & $48 \pm 6.03^{*}$ & $44.96 \pm 7.21^{*}$ & $48.61 \pm 6.14^{*}$ \\
\hline Calf Circumference & $34.51 \pm 3.68^{*}$ & $33.61 \pm 3.3^{*}$ & $33.10 \pm 4.26^{*}$ & $33.95 \pm 3.59^{*}$ \\
\hline Elbow Bone Breadth & $13.6 \pm 1.84^{*}$ & $14.35 \pm 1.53^{*}$ & $11.79 \pm 2.38^{\star}$ & $13.41 \pm 2.69^{*}$ \\
\hline Wrist Bone Breadth & $8.09 \pm 1.33^{*}$ & $8.66 \pm 0.99^{*}$ & $6.94 \pm 1.51^{*}$ & $7.07 \pm 0.8^{*}$ \\
\hline Chest Breadth & $50.18 \pm 5.23^{*}$ & $49.55 \pm 5.34^{*}$ & $49.22 \pm 6.23^{*}$ & $49.32 \pm 4.8^{*}$ \\
\hline Knee Bone Breadth & $18.55 \pm 3.44^{*}$ & $19.46 \pm 1.85^{*}$ & $16.63 \pm 2.36^{*}$ & $17.27 \pm 2^{*}$ \\
\hline Ankle Bone Breadth & $11.88 \pm 1.44^{*}$ & $12.42 \pm 1.43^{*}$ & $10.73 \pm 1.32^{*}$ & $11.01 \pm 0.96^{*}$ \\
\hline Log Chin & $0.982 \pm 0.12^{*}$ & $0.98 \pm 0.14^{*}$ & $1.022 \pm 0.104^{*}$ & $0.99 \pm 0.12^{*}$ \\
\hline Log Biceps & $0.93 \pm 0.20^{*}$ & $0.93 \pm 0.196^{*}$ & $1.07 \pm 0.16^{*}$ & $1.11 \pm 0.11^{\star}$ \\
\hline Log Triceps & $1.13 \pm 0.18^{\star}$ & $1.18 \pm 0.107^{*}$ & $1.24 \pm 0.13^{*}$ & $1.26 \pm 0.15^{\star}$ \\
\hline Log Subscapular & $1.30 \pm 0.19^{*}$ & $1.32 \pm 0.13^{*}$ & $1.38 \pm 0.14^{*}$ & $1.41 \pm 0.8^{*}$ \\
\hline Log Chest & $1.21 \pm 0.24^{*}$ & $1.18 \pm 0.26^{*}$ & $1.38 \pm 0.21^{*}$ & $1.23 \pm 0.4^{*}$ \\
\hline Log Abdominal & $1.41 \pm 0.21^{*}$ & $1.33 \pm 0.19^{*}$ & $1.47 \pm 0.14^{*}$ & $1.43 \pm 0.15^{*}$ \\
\hline Log Suprailiac & $1.25 \pm 0.25^{*}$ & $1.15 \pm 0.19^{*}$ & $1.45 \pm 0.18^{*}$ & $1.4 \pm 0.11^{\star}$ \\
\hline Log Thigh & $1.30 \pm 0.205^{*}$ & $1.32 \pm 0.19^{*}$ & $1.42 \pm 0.155^{\star}$ & $1.3 \pm 0.23^{*}$ \\
\hline Log Knee & $1.00 \pm 0.19^{*}$ & $1.04 \pm 0.17^{*}$ & $1.06 \pm 0.22^{*}$ & $0.97 \pm 0.37^{*}$ \\
\hline Log Calf & $1.21 \pm 0.21^{*}$ & $1.19 \pm 0.18^{*}$ & $1.31 \pm 0.19^{*}$ & $1.31 \pm 0.16^{*}$ \\
\hline
\end{tabular}

'*' Shows that $\mathrm{P}$ value is $<0.001$ which is highly significant for all the variables. The comparison is within a gender i.e. between males of the prediction and validation set and females of the prediction and validation set

Table 2: Descriptive data of prediction set and validation set for both males and females

\begin{tabular}{|c|c|c|c|}
\hline \multicolumn{5}{|c|}{ MALES } \\
\hline Model & R & R Square & Std. Error of the Estimate \\
\hline 1 & 0.95 & 0.90 & 2.85 \\
\hline 2 & 0.84 & 0.71 & 3.24 \\
\hline \multicolumn{7}{|c|}{ FEMALES } \\
\hline 1 & 0.95 & 0.90 & 2.42 \\
\hline 2 & 0.85 & 0.72 & 2.74 \\
\hline
\end{tabular}

Table 3: Model summary for both males and females using correlation between Model 1 and Model 2

\begin{tabular}{|c|c|c|c|c|c|c|}
\hline \multicolumn{7}{|c|}{ MALES } \\
\hline \multirow{4}{*}{1} & MODEL & SUM OF SQUARES & Df & MEAN OF SQUARES & F & SIGNIFICANCE \\
\hline & Regression & 1682.992 & 32 & 52.75 & 6.39 & \multirow[t]{3}{*}{.000} \\
\hline & Residual & 181.629 & 22 & 8.26 & & \\
\hline & Total & 1869.542 & 54 & & & \\
\hline \multirow{3}{*}{2} & Regression & 1325.082 & 2 & 662.54 & 63.218 & \multirow[t]{3}{*}{.000} \\
\hline & Residual & 544.460 & 52 & 10.47 & & \\
\hline & Total & 1869.542 & 54 & & & \\
\hline
\end{tabular}




\begin{tabular}{|c|c|c|c|c|c|c|}
\hline \multicolumn{7}{|c|}{ MALES } \\
\hline & MODEL & SUM OF SQUARES & Df & MEAN OF SQUARES & $F$ & SIGNIFICANCE \\
\hline \multicolumn{7}{|c|}{ FEMALES } \\
\hline \multirow{3}{*}{1} & Regression & 1054.192 & 28 & 37.65 & 6.442 & \multirow[t]{3}{*}{.000} \\
\hline & Residual & 116.894 & 20 & 5.845 & & \\
\hline & Total & 1171.087 & 48 & & & \\
\hline \multirow{3}{*}{2} & Regression & 839.63 & 4 & 209 & 27.865 & \multirow[t]{3}{*}{.000} \\
\hline & Residual & 331.456 & 44 & 7.533 & & \\
\hline & Total & 1171.087 & 48 & & & \\
\hline
\end{tabular}

Table 4: Analysis of variance for predicted models with hydrodensitometry as a dependent variable for both males and females

\section{Models for males are}

MODEL 1: BODY FAT \% = - $71.28-0.25$ (AGE) - 29.40 (HEIGHT) + 0.35 (WEIGHT) + 4.15 (SKF SUBSCAPULAR) - 0.67 $($ CHEST SKF $)+0.306($ THIGH SKF $)+5.13($ KNEE SKF $)-1.01($ CALF SKF $)-1.01$ (ARM CIRCUMFERENCE) + $0.38($ HIP CIRCUMFERENCE) - 0.39 (CALF CIRCUMFERENCE) - 28.05 (LOG TRICEPS) - 145.87 (LOG SUBSCAPULAR) - 45.58 (LOG ABDOMINAL) - 24.86 (LOGSUPRAILIAC) - 25.29 (LOGTHIGH) - 92.325 (LOG KNEE) + 0.032 (CHIN SKF) ${ }^{2}-0.118$ (BICEPS SKF)2 - $0.174(\text { TRICEPS SKF })^{2}-0.101\left(\right.$ SUBSCAPULAR SKF) ${ }^{2}+0.01\left(\right.$ CHEST SKF) $2+0.005\left(\right.$ ABDOMINAL SKF) ${ }^{2}-0.015$ $(\text { SUPRAILIAC SKF })^{2}-0.074(\text { KNEE SKF })^{2}+0.020(\text { CALF SKF })^{2}-0.01\left(W^{\star} \mathrm{H}\right)+154.64($ LOG7 $)+104.37($ LOG4) +0.127 $\left(\mathrm{BI}^{\star} \mathrm{TRI}\right)+0.162\left(\mathrm{TRI}^{\star} \mathrm{SS}\right)$.

Where skf is skin folds, $\left(\mathrm{W}^{\star} \mathrm{H}\right)$ is a product of waist circumference and hip circumference in $\mathrm{cm},(\log 4)$ includes sum of skin folds of biceps, triceps, subscapular, suprailiac in $\mathrm{mm}$ with $\log$ base $10,(\log 7)$ includes sum of skin folds of biceps, triceps, subscapular, abdominal, suprailiac, thigh, calf in mm with log base $10,\left(\mathrm{TRI}{ }^{\star} \mathrm{SS}\right.$ ) is a product of skin fold of triceps and subscapular in mm. (SI * SS) is a product of subscapular and suprailiac in $\mathrm{mm}$.

MODEL 2: BODY FAT \% = -27.245 + 32.43 (LOG 5), where LOG 5 is a sum of skin folds of biceps, triceps, subscapular, suprailiac and calf in $\mathrm{mm}$ with $\log$ base 10 . Unstandardized coefficient for (suprailiac skf) ${ }^{3}$ was very low i.e. $-4.94 \mathrm{E}-005$ and was therefore not included in the model.

\section{Models for females are}

MODEL 1: BODY FAT \% = 1.844 + 0.18 (AGE) + 33.59 (HEIGHT) $-0.14($ WEIGHT $)+1.476($ CHEST SKF $)-2.22($ THIGHSKF $)+$ 0.144 (KNEESKF) - 0.411 (ARM CIRCUMFERENCE) + 0.48 (WAIST CIRCUMFERENCE) + 1.448 (CALFCIRCUMFERENCE) + $124.48($ LOGCHIN) + 42.6 (LOG TRICEPS) + 60.78 (LOG SUBSCAPULAR) - 63.54 (LOG CHEST) + 44.08 (LOG ABDOMINAL) +64.6 (LOG SUPRAILIAC) - $0.185(\text { CHIN SKF) })^{2}-0.025(\text { BICEPS SKF })^{2}-0.006(\text { TRICEPS SKF })^{2}-0.008\left(\right.$ CHEST) ${ }^{2}-0.008$ $(\text { ABDOMINAL SKF })^{2}-0.016(\text { SUPRAILIAC SKF })^{2}+0.036(\text { THIGH SKF })^{2}-.003\left(\mathrm{~W}^{\star} \mathrm{H}\right)+0.027\left(\mathrm{BI}^{\star} \mathrm{TRI}\right)+0.029\left(\mathrm{SI}{ }^{\star} \mathrm{SS}\right)-0.016$ $\left(\mathrm{SS}^{\star} \mathrm{AB}\right)+0.018\left(\mathrm{AB}^{\star} \mathrm{SI}\right)-209.839$ (LOG3). Where LOG 3 is the sum of triceps, subscapular and suprailiac skinfolds in mm with the $\log$ base $10 .\left(\mathrm{SS}^{\star} \mathrm{AB}\right)$ is a product of subscapular skf and abdominal skf in $\mathrm{mm},\left(\mathrm{AB} \mathrm{B}^{\star} \mathrm{SI}\right)$ is a product of abdominal skf and suprailiac Skinfolds.

MODEL 2: BODY FAT $\%=-13.71+1.26($ ARM $)-0.014\left(\mathrm{BI}^{\star}\right.$ TRI $)+10.58($ LOG ABDOMINAL SKF $)+0.002(\text { CHEST SKF })^{2}$ where $\left(\mathrm{BI}{ }^{*} \mathrm{TRI}\right)$ is a product of skin fold of biceps and triceps in $\mathrm{mm}$.

\section{Comparison of new predicted equations with hydrodensitometry, DEXA and other existing skin fold equations}

The results of body fat \% calculated from hydro densitometry, DEXA, existing equations and new predicted equations are shown in Table 5A. In the new predicted equations both model 1 (males $33.24 \pm 6.8$ and females $36.11 \pm 7$ ) and model 2 (males $34.13 \pm$ 5.09 and females $35.57 \pm 3.7$ ) are closely related to hydrodensitometry (males $33.21 \pm 5.85$ and females $35.99 \pm 4.9$ ), which is the gold standard for body fat \% estimation. The error differences in model 1 (for males $-0.039 \pm 5.14$ and for females $-0.42 \pm 4.03$ ) and in model 2 (for males $-0.93 \pm 3.88$ and females $-0.121 \pm 7.05$ ) are also comparatively lower for both males and females shown in Table 5B. Based on our results, we found that model 1 and 2 are most closely related to hydro densitometry followed by DEXA, DWW, JP 3, JP 4, Garcia, and BMI bf \% in males and DWW, BMI bf \%, DEXA, JP 4, JP 3 and Garcia in females.

Tables 6A and 6B show the mean values and standard deviations of BMI, BMD, WHR and \% BF derived from selected models classified according to BMI categories in males and females respectively. They clearly show that the newly developed models give a higher value of percent body fat than previously used equations. Further in females as percent body fat increases the BMD tends to increase. 


\begin{tabular}{|c|c|c|}
\hline BF \% & $\begin{array}{c}\text { MALES }(\mathbf{N}=\mathbf{7 7}) \\
\text { Mean } \pm \text { SD }(\mathbf{C I} \text { at 95\%) }\end{array}$ & $\begin{array}{c}\text { FEMALES }(\mathbf{N}=\mathbf{6 0}) \\
\text { Mean } \pm \text { SD }(\mathbf{C I} \text { at 95\%) }\end{array}$ \\
\hline Hydrodensiometry & $33.21 \pm 5.85(17.14,41.36)$ & $35.99 \pm 4.9(21.13,44.45)$ \\
\hline DEXA & $30.31 \pm 5.87(13.10,42.10)$ & $41.25 \pm 5.85(19.1,53.5)$ \\
\hline DWW & $26.74 \pm 5.47(9.55,37.40)$ & $36.67 \pm 4.30(20.7,43.1)$ \\
\hline JP3 & $21.33 \pm 7.33(4.94,37.10)$ & $29.89 \pm 5.25(11.97,39.7)$ \\
\hline JP4 & $21.60 \pm 6.35(5.43,35.80)$ & $30.32 \pm 5.04(12.4,38.36)$ \\
\hline BMI Body fat \% & $58.38 \pm 10.34(38.71,77.16)$ & $36.46 \pm 6.87(3.34,47.15)$ \\
\hline Garcia Equation & $21.82 \pm 6.8(-1.81,36.22)$ & $55.38 \pm 119.65$ \\
& $33.24 \pm 6.8(5.94,54.24)$ & $36.11 \pm 7(21.4,57.6)$ \\
\hline Model 1 & $34.13 \pm 5.09(14.73,44.15)$ & $35.57 \pm 3.7(22.28,43.78)$ \\
\hline Model 2
\end{tabular}

Table5A: BF \% Estimation by Hydrodensitometery, DEXA, existing equations and new predicted equations

\begin{tabular}{|c|c|c|}
\hline Error Differences & $\begin{array}{c}\text { MALES } \\
\text { Mean } \pm \text { SD }(\text { CI at 95\%) }\end{array}$ & $\begin{array}{c}\text { FEMALES } \\
\text { Mean } \pm \text { SD }(\text { CI at 95\%) }\end{array}$ \\
\hline Hydrodensitometry-DEXA & $2.8 \pm 4.22(-9.73,9.92)$ & $-3.94 \pm 9.45(-21.27,37.23)$ \\
\hline Hydrodensitometry-DWW & $6.47 \pm 4.71(-6.86,16.99)$ & $-0.68 \pm 4.77(-18.47,8.88)$ \\
\hline Hydrodensitometry-JP 3 & $11.88 \pm 6.14(-4.10,28.66)$ & $6.11 \pm 5.43(-4.87,19.32)$ \\
\hline Hydrodensitometry-JP 4 & $11.6 \pm 5.25(-2.22,24.9)$ & $5.67 \pm 5.18(-5.84,17.55)$ \\
\hline Hydrodensitometry-BMI body fat \% & $-25.18 \pm 10.44(-51.30,-7.21)$ & $-0.47 \pm 5.8(-16.11,23.14)$ \\
\hline Hydrodensitometry-Garcia & $11.39 \pm 4.98(1.53,21.39)$ & $-19.32 \pm 118.9(-922.62,27.19)$ \\
\hline Hydrodensitometry-Model1 & $-0.039 \pm 5.14(-14.56,26.54)$ & $0.42 \pm 4.03(-11.79,11.40)$ \\
\hline Hydrodensitometry-Model2 & $-0.93 \pm 3.88(-12.55,6.42)$ & $-0.121 \pm 7.05(-21.46,17.41)$ \\
\hline
\end{tabular}

Table 5B: Standard Error Differences between Hydrodensitometry, DEXA, Existing Equations and New Predicted Equations

\begin{tabular}{|c|c|c|c|c|c|c|c|c|c|c|}
\hline VARIABLES & BMI & $\begin{array}{l}\text { BMD } \\
\mathrm{g} / \mathrm{cm}^{2}\end{array}$ & WHR & $\begin{array}{l}\text { UWW } \\
\% \text { BF }\end{array}$ & $\begin{array}{c}\text { DWW } \\
\% \mathrm{BF}\end{array}$ & $\begin{array}{l}\text { JP3 } \\
\% \text { BF }\end{array}$ & $\begin{array}{c}\text { JP4 } \\
\% \text { BF }\end{array}$ & $\begin{array}{c}\text { DEXA } \\
\% \text { BF }\end{array}$ & $\begin{array}{c}\text { MODEL1 } \\
\% \mathrm{BF}\end{array}$ & $\begin{array}{c}\text { MODEL } 2 \\
\% \mathrm{BF}\end{array}$ \\
\hline $\begin{array}{c}\mathrm{BMI}<23 \\
\mathrm{~N}=13\end{array}$ & $\begin{array}{c}21.14 \pm \\
2.06\end{array}$ & $\begin{array}{c}1.12 \pm \\
0.07\end{array}$ & $\begin{array}{c}0.94 \pm \\
0.07\end{array}$ & $\begin{array}{c}27.03 \pm \\
6.62\end{array}$ & $\begin{array}{c}21.27 \pm \\
5.14\end{array}$ & $\begin{array}{c}13.80 \pm \\
4.63\end{array}$ & $\begin{array}{c}15.18 \pm \\
4.21\end{array}$ & $\begin{array}{c}23.95 \pm \\
5.20\end{array}$ & $\begin{array}{c}27.89 \pm \\
5.59\end{array}$ & $\begin{array}{c}28.69 \pm \\
5.48\end{array}$ \\
\hline $\begin{array}{c}\text { BMI 23-27.9 } \\
\mathrm{N}=43\end{array}$ & $\begin{array}{c}25.95 \pm \\
1.38\end{array}$ & $\begin{array}{c}1.14 \pm \\
0.11\end{array}$ & $\begin{array}{c}0.97 \pm \\
0.06\end{array}$ & $\begin{array}{c}33.33 \pm \\
5.30\end{array}$ & $\begin{array}{c}26.98 \pm \\
5.18\end{array}$ & $\begin{array}{c}21.13 \pm \\
6.89\end{array}$ & $\begin{array}{c}21.14 \pm \\
5.72\end{array}$ & $\begin{array}{c}29.95 \pm \\
4.95\end{array}$ & $\begin{array}{c}32.46 \pm \\
6.64\end{array}$ & $\begin{array}{c}33.79 \pm \\
4.14\end{array}$ \\
\hline $\begin{array}{c}\mathrm{BMI}>28 \\
\mathrm{~N}=21\end{array}$ & $\begin{array}{c}30.37 \pm \\
1.90\end{array}$ & $\begin{array}{c}1.13 \pm \\
0.06\end{array}$ & $\begin{array}{c}1.01 \pm \\
0.06\end{array}$ & $\begin{array}{c}36.77 \pm \\
2.58\end{array}$ & $\begin{array}{c}29.62 \pm \\
3.65\end{array}$ & $\begin{array}{c}26.39 \pm \\
5.28\end{array}$ & $\begin{array}{c}26.53 \pm \\
4.60\end{array}$ & $\begin{array}{c}34.95 \pm \\
35.81\end{array}$ & $\begin{array}{c}38.18 \pm \\
4.47\end{array}$ & $\begin{array}{c}38.20 \pm \\
2.79\end{array}$ \\
\hline
\end{tabular}

Table 6A: Mean values and standard deviations of BMI, BMD, WHR and \% BF derived from selected models classified according to BMI categories in males

\begin{tabular}{|c|c|c|c|c|c|c|c|c|c|c|}
\hline VARIABLES & BMI & $\begin{array}{l}\text { BMD } \\
\mathrm{g} / \mathrm{cm}^{2}\end{array}$ & WHR & $\begin{array}{l}\text { UWW } \\
\% \text { BF }\end{array}$ & $\begin{array}{c}\text { DWW } \\
\% \text { BF }\end{array}$ & $\begin{array}{c}\text { JP3 } \\
\% \text { BF }\end{array}$ & $\begin{array}{c}\text { JP4 } \\
\% \text { BF }\end{array}$ & $\begin{array}{c}\text { DEXA } \\
\% \text { BF }\end{array}$ & $\begin{array}{c}\text { MODEL1 } \\
\% \mathrm{BF}\end{array}$ & $\begin{array}{c}\text { MODEL } 2 \\
\% \mathrm{BF}\end{array}$ \\
\hline $\begin{array}{c}\mathrm{BMI}<23 \\
\mathrm{~N}=10\end{array}$ & $\begin{array}{c}21.42 \pm \\
1.83\end{array}$ & $\begin{array}{c}1.06 \pm \\
0.08\end{array}$ & $\begin{array}{c}0.89 \pm \\
0.04\end{array}$ & $\begin{array}{c}30.80 \pm \\
4.30\end{array}$ & $\begin{array}{c}32.06 \pm \\
5.31\end{array}$ & $\begin{array}{c}25.18 \pm \\
6.79\end{array}$ & $\begin{array}{c}26.18 \pm \\
6.91\end{array}$ & $\begin{array}{c}35.88 \pm \\
6.28\end{array}$ & $\begin{array}{c}31.78 \pm \\
6.72\end{array}$ & $\begin{array}{c}31.57 \pm \\
4.29\end{array}$ \\
\hline $\begin{array}{c}\text { BMI 23-27.9 } \\
\text { N = 27 }\end{array}$ & $\begin{array}{c}25.62 \pm \\
1.46\end{array}$ & $\begin{array}{c}1.08 \pm \\
0.14\end{array}$ & $\begin{array}{c}0.93 \pm \\
0.06\end{array}$ & $\begin{array}{c}36.01 \pm \\
4.40\end{array}$ & $\begin{array}{c}36.90 \pm \\
3.33\end{array}$ & $\begin{array}{c}30.38 \pm \\
3.97\end{array}$ & $\begin{array}{c}31.22 \pm \\
4.08\end{array}$ & $\begin{array}{c}40.11 \pm \\
5.12\end{array}$ & $\begin{array}{c}36.70 \pm \\
6.29\end{array}$ & $\begin{array}{c}35.27 \pm \\
2.78\end{array}$ \\
\hline $\begin{array}{c}\mathrm{BMI}>28 \\
\mathrm{~N}=23\end{array}$ & $\begin{array}{c}30.87 \pm \\
2.08\end{array}$ & $\begin{array}{c}1.09 \pm \\
0.08\end{array}$ & $\begin{array}{c}0.92 \pm \\
0.05\end{array}$ & $\begin{array}{c}38.24 \pm \\
4.05\end{array}$ & $\begin{array}{c}38.41 \pm \\
3.47\end{array}$ & $\begin{array}{c}31.36 \pm \\
4.89\end{array}$ & $\begin{array}{c}31.07 \pm \\
4.42\end{array}$ & $\begin{array}{c}45.21 \pm \\
3.84\end{array}$ & $\begin{array}{c}37.31 \pm \\
7.47\end{array}$ & $\begin{array}{c}37.67 \pm \\
2.85\end{array}$ \\
\hline
\end{tabular}

Table 6B: Mean values and standard deviations of BMI, BMD, WHR and \% BF derived from selected models classified according to BMI categories in females 


\section{Discussion}

The assessment of body composition to mark obesity becomes very important to rule out diseases related to body fatness [19]. All popular skinfold thickness equation models currently in use, were derived and validated using data obtained from diverse populations, but did not use data primarily of Asian Indians. In this study we evaluated data from several sources including DEXA and kinanthropometric parameters using hydrodensitometry as the gold standard.

The anthropometric parameters used to assess body fat included, skinfold thickness, BMI and waist to hip ratio, an indicator of intra-abdominal obesity [19]. The sites of skin folds we used to calculate body fat, included biceps, triceps, subscapular, chest, abdominal, suprailiac, thigh, knee and calf. Circumferences (arm, waist, hip, thigh and calf), bone breadth (elbow, wrist, chest, knee and ankle) ,body density using hydrodensitometry was calculated by mass/volume which is a function of additive densities of four components including fat and those of fat free body consisting of water, mineral and residual components (which consist mostly of protein). Using key variables, which were shown to be significantly associated with body fat \% derived from hydrodensitometry, with the help of linear regression models, we developed a prediction equation in a wide age group of Asian Indians i.e. from 30-60 years of age.

The newly developed skin fold equation is important for 2 reasons: firstly, it was validated and cross validated using hydrodensitometry as a gold standard and secondly, no previous studies have been conducted in a wide age group of Asian Indians. It is clear from our results that model 1 is very accurate and is best for deriving a very close estimation of body fat \%. If investigators desire a highly precise body fat estimation with its $\mathrm{R}^{2}$ of 0.90 and 0.917 for males and females respectively, they can use model 1 . However this clearly entails measurements from 20 physical characteristics. Considering economy of time and convenience, model 2 , which entails the use of only 3 and 5 physical characteristics, in males and females respectively, is recommended. This model 2 , with an accuracy of $\mathrm{R}^{2}$ of 0.71 and $\mathrm{R}^{2}$ of 0.84 for males and females respectively, in fact is greater than the $\mathrm{R}^{2}$ values we obtained for our population using DEXA and other existing skin fold equations. After cross validating model 2, we compared the values of body fat $\%$ obtained using this equation, with popular skin fold prediction equations, the Durnin and Womersley equation, Jackson and Pollock 3 site and 4 site equations, Garcia equation, BMI body fat \% equations, body fat \% calculated by DEXA. We found that the $\mathrm{R}$ value of our model 2 with hydrodensitometry was more than all existing skin fold equation and DEXA.

Though it was not our primary aim to evaluate BMD, we observed a positive relation between BMI and BMD in women. We also found that the BMD of our subjects for comparable age group in Caucasians were much lower [20]. Earlier studies have indicated that there was a positive relationship; i.e. as body fat \% increased, BMD also increased [21-27] and in females this observation held true. This was attributed to the fact that with an increase in body weight, the mechanical loading on bones would increase [20].

A limitation of this study was that this study was a single centered trial and its validation in other geographical areas needs to be studied. This could not be accomplished because to date there is no other center in India, which utilizes hydrodensitometry for the estimation of body fat. Another limitation is that we did not take into account menopausal status of women, which would affect body fat accumulation. However, we desired to formulate an equation, which would suit a broad age group of women. Future work in this area could take into account menopausal status, which could be incorporated into the equation. Further it is important to mention here that in our study we had great difficulty in getting volunteers to participate in the process of underwater weighing for cultural as well as other reasons, which included a phobia of staying underwater without breathing, restriction in range of motion at hip and knee. Almost $40 \%$ of those who initially volunteered had to be finally excluded because they were unable to perform complete exhalation and then stay underwater for the required time.

Given the difficulties associated with hydrodensitometry many investigators now use DEXA, which is based on the 4-compartment model for the calculation of body fat percentage and bone mineral density, given its non-invasive nature as well as convenience. However in DEXA [28-32] body fat is calculated on the constancy assumption that $\approx 73 \%$ of lean body mass (lean + BMC) is water [29-32]. However the tissue water content in adipose tissue is highly variable e.g. ranging from $\pm 17 \%$ to $50 \%$ in humans [30-32], which can thus affect the accuracy of the result. Despite these limitations, the relatively better accuracy of the equation developed by us demonstrates the need for the development of population specific equations for the assessment of body composition.

\section{Conclusion}

We developed a new prediction equation for assessing the total body fat by using hydrodensitometry as the gold standard method to assess body composition with anthropometric measurements. Further validation and cross validation revealed that the new model was more significant, as its error difference is less as compare to the existing models. Further validation of this equation is recommended.

\section{Acknowledgement}

The authors would like to acknowledge and profoundly thank Prof S K Neogy, Head SQCN and OR Unit, Indian Statistical Institute, New Delhi for his whole hearted assistance in the statistical analysis, and the volunteers for their co-operation and participation in the study. 


\section{References}

1. WHO expert consultation (2004) Appropriate body mass index for Asian populations and its implications for policy and intervention strategies. Lancet 363: $157-63$.

2. Whaley MH, Brubaker PH, Otto RM, Armstrong LE (2006) American College of Sports Medicine ACSM’s Guidelines for Exercise Testing and Prescription (7th Edn) Philadelphia(PA): Lipincott Williams and Wilkins Publishers, USA.

3. WHO Expert Consultation (2004) Appropriate body mass index for Asian populations and its implications for policy and intervention strategies. Lancet 363: $157-63$.

4. Okorodudu DO, Jumean MF, Montori VM, Romero-Corral A, Somers VK, et al. (2010) Diagnostic performance of body mass index to identify obesity as defined by body adiposity: a systematic review and meta-analysis. Int J Obes 34: 791-9.

5. Zeng Q, Dong SY, Sun XN, Xie J, Cui Y (2012) Percent body fat is a better predictor of cardiovascular risk factors than body mass index. Braz J Med Biol Res 45: 591-600.

6. Appel SJ, Jones ED, Kennedy-Malone L (2004) Central obesity and the metabolic syndrome: implications for primary care providers. J Am Acad Nurse Pract 16: $335-42$.

7. Harvie M, Hooper L, Howell AH (2003) Central obesity and breast cancer risk: a systematic review. Obes Rev 4: 157- 73.

8. Peterson MJ, Czerwinski SA, Siervogel RM (2003) Development and validation of skinfold - thickness prediction equations with a 4 - compartmental model 1-3. Am J Clin Nutr 77: 1186-91.

9. Nevill AM, Metsios GS, Jackson AS, Wang J, Thornton J, et al. (2008) Can we use the Jackson and Pollock equations to predict body density/fat of obese individuals in the 21st century? Int J Body Compos Res 6: 114-21.

10. Immink MD, Flores R, Diaz EO (1992) Body mass index, body composition and the chronic energy deficiency classification of rural adult populations in Guatemala. Eur J Clin Nutr 46: 419-27.

11. Behnke AR, Feen BG, Welham WC (1942) The Specific Gravity of Healthy Men Body Weight $\div$ Volume as an Index of Obesity. JAMA 118: 495-8.

12. Dempster P, Aitkens S (1995) A new air displacement method for the determination of human body composition. Med Sci Sports Exerc 27: 1692-7.

13. Lohman TG, Roche AF, Martorell R (1988) Anthropometric standardization reference manual. IL: Human Kinetic Books, Champaign Publisher, USA.

14. Durnin JV, Womersley J (1974) Body fat assessed from total body density and its estimation from skinfold thickness: measurements on 481 men and women aged from 16 to 72 years. Br J Nutr 32: 77-97.

15. Brozek J, Grande F, Anderson JT, Keys A (1963) Densito- metric analysis of body composition: revision of some quantitative assumptions. Ann N Y Acad Sci 110: $113-40$.

16. Sharp DS, Andrew ME, Burchfiel CM, Violanti JM, Wactawski-Wende J (2012) Body mass index versus dual energy x-ray absorptiometry-derived indexes: predictors of cardiovascular and diabetic disease risk factors. Am J Hum Biol 24: 400-5.

17. Visser M, Fuerst T, Lang T, Salamone L, Harris TB (1999) Validity of fan-beam dual-energy X-ray absorptiometry for measuring fat-free mass and leg muscle mass. Health, Aging, and Body Composition Study--Dual-Energy X-ray Absorptiometry and Body Composition Working Group. J Appl Physiol 87: 1513-20.

18. Fantin F, Di Francesco V, Fontana G, Zivelonghi A, Bissoli L, et al. (2007) Longitudnal body composition changes in old men and women: interrelationships with worsening disability. J Gerontol A Biol Sci Med Sci 62: 1375-81.

19. He M, Tan KC, Li ET, Kung AW (2001) Body fat determination by dual energy X-ray absorptiometry and its relation to body mass index and waist circumference in Hong Kong Chinese. Int J Obes Relat Metab Disord 25:748-52.

20. Ross PD, He Y, Yates AJ, Coupland C, Ravn P, et al. (1996) Body size accounts for most differences in bone density between Asian and Caucasian women. The EPIC (Early Postmenopausal Interventional Cohort) Study Group. Calcif Tissue Int 59: 339-43.

21. Castro JP, Joseph LA, Shin JJ, Arora SK, Nicasio J, et al. (2005) Differential effect of obesity on bone mineral density in White,Hispanic and African American women: a cross sectional study. Nutr Metab 2: 9.

22. Aloia JF, McGowan DM, Vaswani AN, Ross P, Cohn SH (1991) Relationship of menopause to skeletal and muscle mass. Am J Clin Nutr 53: 1378-83.

23. Albala C, Yáñez M, Devoto E, Sostin C, Zeballos L, et al. (1996) Obesity as a protective factor for postmenopausal osteoporosis. Int J Obes Metab Disord 20: $1027-32$.

24. Chen Z, Lohman TG, Stini WA, Ritenbaugh C, Aickin M (1997) Fat or lean tissue mass: which one is the major determinant of bone mineral mass in healthy postmenopausal women? J Bone Miner Res 12: 144-51.

25. Felson DT, Zhang Y, Hannan MT, Anderson JJ (1993) Effects of weight and body mass index on bone mineral density in men and women: the Framingham study. J Bone Miner Res 8: 567-73.

26. Khosla S, Atkinson EJ, Riggs BL, Melton LJ III (1996) Relationship between body composition and bone mass in women. J Bone Miner Res 11: 857-63.

27. El Maghraoui A, Guerboub AA, Achemlal L, Mounach A, Nouijai A, et al. (2006) Bone mineral density of the spine and femur in healthy Moroccan women. J Clin Densitom 9: 454-60.

28. Müller MJ, Bosy-Westphal A, Kutzner D, Heller M (2003) Metabolically active components of fat free mass (FFM) and resting energy expenditure (REE) in humans. Forum Nutr 56: 301-3.

29. Guo Y, Franks PW, Brookshire T, Antonio Tataranni P (2004) The intra- and inter-instrument reliability of DXA based on ex vivo soft tissue measurements. Obes Res 12: 1925-9.

30. Clarys JP, Scafoglieri A, Provyn S, Louis O, Wallace JA, et al. (2010) A macro-quality evaluation of DXA variables using whole dissection, ashing, and computer tomography in pigs. Obesity $18:$ 1477-85.

31. Provyn S, Clarys JP, Wallace J, Scafoglieri A, Reilly T (2008) Quality control, accuracy, and prediction capacity of dual energy X-ray absorptiometry variables and data acquisition. J Physiol Anthropol 27: 317-23.

32. Scafoglieri A, Provyn S, Wallace J, Louis O, Tresignie J, et al. (2011) Whole body composition by Hologic QDR 4500/A DEXA: system Reliability Versus user Accuracy and precision, Croatia. 


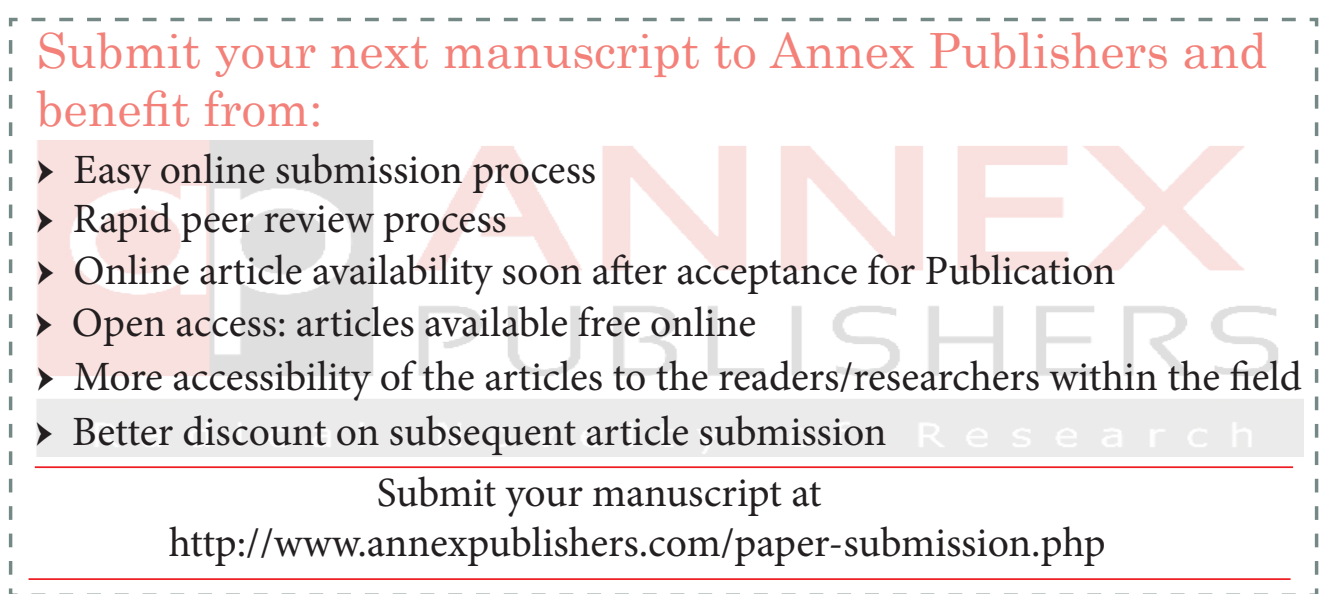

